\title{
Enfermedad por IgA lineal del adulto
}

\section{Linear IgA disease of adults}

\author{
Josefina de Peña-Ortiz, * Maribet González-González,; Denisse Herrera-Bringas ${ }^{\S}$
}

\section{RESUMEN}

La enfermedad por IgA lineal es una dermatosis autoinmune poco frecuente, caracterizada clínicamente por la presencia de ampollas de contenido seroso o serohemático, tensas, que producen prurito en grado variable. Su evolución y pronóstico es distinto en niños y adultos. En este artículo presentamos el caso de una paciente adulta con lesiones muy diseminadas, que tuvo buena respuesta con el tratamiento con dapsona y prednisona.

Palabras clave: IgA lineal, ampollas de contenido seroso, dapsona.
ABSTRACT

Linear IgA bullous dermatosis is a rare, autoimmune dermatosis, clinically characterized by the presence of blisters with serous or serohematic fluid, strained, with variable pruritus degrees. The course and prognosis are different in children and adults. In this article we present the case of an adult female patient, with disseminated disease, and good response to treatment with dapsone and prednisone.

Keywords: Linear IgA, blisters with serous fluid, dapsone.

\section{INTRODUCCIÓN}

La enfermedad por IgA lineal, también conocida como «Dermatosis bulosa por IgA líneal», es una dermatosis ampollosa poco frecuente, autoinmune y adquirida, caracterizada a nivel ultraestructural por el depósito de IgA lineal a lo largo de la unión dermoepidérmica, evidente por inmunofluorescencia directa. $^{1}$

\section{Historia}

La primera descripción de la enfermedad por IgA lineal la realizó Bowen en 1901 como una variante de la dermatitis herpetiforme. ${ }^{2}$ Fue hasta 1979 cuando Jablonska y colaboradores la reconocieron como una entidad diferente debido a los hallazgos clínicos y por inmunohistoquímica. $^{3}$

\footnotetext{
* Dermatóloga.

‡ Dermatopatóloga.

$\S$ Residente del tercer año de Dermatología.
}

Centro Dermatológico «Dr. Ladislao de la Pascua», SSCDMX.

\section{Epidemiología}

Se calcula una incidencia de 0.2-2.3 por 1,000,000 de habitantes por año. ${ }^{4}$ En Europa se incrementa hasta un 0.5 por $1,000,000$ habitantes. Países como China y otros en Asia publican una frecuencia aún mayor.

Afecta tanto a niños como a adultos con dos picos de incidencia: el primero en la infancia temprana, y el segundo en pacientes mayores de 60 años. ${ }^{5}$

\section{Fisiopatología}

Existen diversas teorías sobre los posibles factores que propician la producción de autoanticuerpos IgA, desde infecciones producidas por el virus varicela zóster, autoinmunidad, antecedente de ingesta de medicamentos como vancomicina, ampicilina y ceftriaxona, y la presencia de tumores malignos.

La predisposición genética (en el caso de los pacientes pediátricos) tiene un papel importante. En ellos se han identificado algunos haplotipos HLA

Citar como: de Peña-Ortiz J, González-González M, Herrera-Bringas D. Enfermedad por IgA lineal del adulto. Rev Cent Dermatol Pascua. 2020; 29 (2-3): 81-85. https:// dx.doi.org/10.35366/99920 
específicos como el HLA-B8, HLA-DR3, HLA-DQ2 y HLA-CW7. ${ }^{4}$

Los hallazgos ultraestructurales demuestran la presencia de autoanticuerpos IgA dirigidos contra las proteínas de la membrana basal (derivadas del antígeno BP180) contra desmocolina 1 y anticuerpos IgA anti-Dsg-1 o anti-Dsg3, ${ }^{6}$ los cuales se depositan en forma lineal.

La inmunidad celular también se encuentra alterada, ya que los linfocitos T CD4 se activan provocando el reclutamiento de neutrófilos y eosinófilos que liberan citocinas proinflamatorias, perpetuando la enfermedad.

\section{CUADRO CLÍNICO}

La enfermedad por IgA lineal se caracteriza clínicamente por la aparición gradual o súbita de ampollas de contenido seroso, tensas, sobre una piel eritematosa, lo cual ocasiona prurito de grado variable. Las lesiones en adultos predominan en tronco, extremidades superiores y glúteos. ${ }^{7}$ Suele cursar con exacerbaciones y remisiones, por lo que se le considera una enfermedad persistente. Por el contrario, en los niños el inicio es en general súbito; el padecimiento sigue un curso benigno, y se autolimita. ${ }^{3}$ Específicamente en este grupo etario se describe la presencia de ampollas que confluyen en forma anular, dando la imagen en "Collar de perlas». ${ }^{7}$

En una revisión de 2013, Chanal y colaboradores encontraron que la enfermedad inducida por fármacos se manifiesta con ampollas de mayor tamaño. En estos casos es frecuente observar el signo de Nikolsky positivo, con todo lo que éste representa, debido a que la evolución del padecimiento es más grave en estos pacientes. ${ }^{1}$

\section{Diagnóstico}

Se basa en las características de topografía y morfología clásicas de la dermatosis, y por los hallazgos del estudio histopatológico. En los cortes teñidos con hematoxilina y eosina se observa una ampolla subepidérmica con presencia de neutrófilos y ocasionalmente eosinófilos. El diagnóstico se confirma solicitando la realización de inmunofluorescencia directa de la biopsia de piel, la cual pone en evidencia el depósito de IgA lineal en la unión dermoepidérmica. La inmunofluorescencia indirecta, positiva en 30-50\% de los casos, muestra la presencia de autoanticuerpos circulantes tipo IgA contra la membrana basal dermoepidérmica. ${ }^{8}$

\section{Tratamiento}

El tratamiento de elección en las formas espontáneas es la administración de dapsona a dosis de 1-2 mg/kg/día, con vigilancia estrecha de los pacientes por el riesgo de desarrollar efectos adversos, dentro de los cuales tenemos hemólisis, agranulocitosis, metahemoglobinemia, hepatitis medicamentosa, rash y otros menos graves, aunque frecuentes como cefalea, síntomas gastrointestinales y neuropatía periférica.

Cuando la enfermedad se encuentra muy diseminada, se puede agregar prednisona a dosis de $15-30 \mathrm{mg} / \mathrm{día}$.

Otras opciones terapéuticas descritas con resultados variables son el empleo de inmunoglobulina $G$ intravenosa a dosis de $2-4 \mathrm{~g} / \mathrm{kg}$ por ciclo cada cuatro semanas y sulfapiridina de 1-1.5 g/día. ${ }^{9}$

\section{CASO CLÍNICO}

Se trata de una paciente de 47 años de edad, originaria de la Ciudad de México, con antecedentes de intolerancia a los carbohidratos.

Acude a la consulta externa de dermatología por un padecimiento de 10 meses de evolución, caracterizado por la presencia de «ampollas y comezón en el cuerpo», a decir de la paciente, por lo que acudió a la consulta privada de un dermatólogo, quien la diagnostica como pénfigo, e indica manejo con prednisona $50 \mathrm{mg} / \mathrm{día}$, logrando remisión parcial de la dermatosis a los dos meses de tratamiento. Sin embargo, al suspender el mismo, presenta exacerbación de la enfermedad, motivo por el cual acude al Centro Dermatológico «Dr. Ladislao de la Pascua».

A la exploración física se observa una dermatosis diseminada, con tendencia a la generalización, respetando piel cabelluda, palmas y plantas (Figura 1), constituida por vesículas y ampollas tensas de contenido seroso de 3-8 mm, costras sanguíneas y hemáticas así como manchas residuales (Figura 2) que confluyen formando algunas placas. Evolución crónica y pruriginosa.

Se realiza biopsia de una de las vesículas, y con tinción de rutina se observó una ampolla subepidérmica (Figura 3) con restos de fibrina en el interior y presencia de polimorfonucleares (Figura 4), por lo que se solicita inmunofluorescencia e inmunohistoquímica para IgA e IgG (Figura 5), las cuales fueron reportadas como positivas, concluyendo que se trata de una enfermedad por $\lg$ A lineal.

Se inicia tratamiento con prednisona $75 \mathrm{mg} / \mathrm{día}$, dapsona 100 mg/día, metformina 850 mg/día, emoliente 

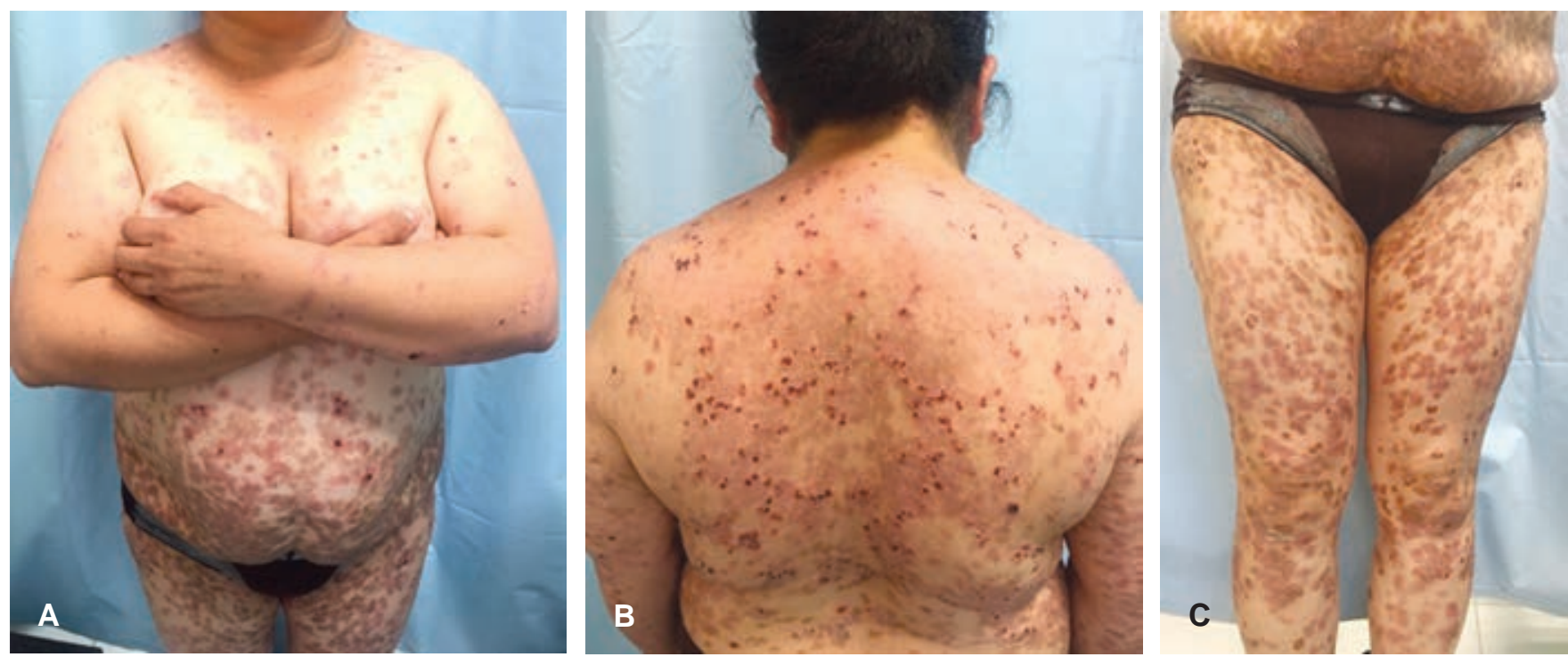

Figura 1: A-C) Aspecto general de la dermatosis.

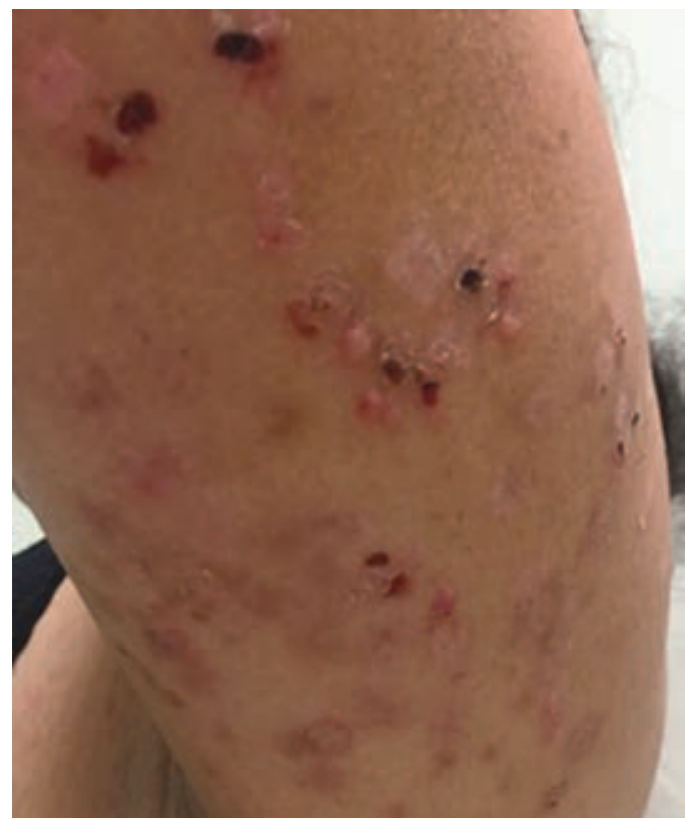

Figura 2: A mayor detalle se observa una ampolla de contenido seroso, exulceraciones, eritema y manchas residuales.

y filtro solar con horario y se solicitan exámenes de laboratorio de control.

La dosis de prednisona se fue disminuyendo en forma gradual hasta suspenderla.

La paciente evolucionó en forma favorable, y cinco meses después de haber iniciado el tratamiento acude

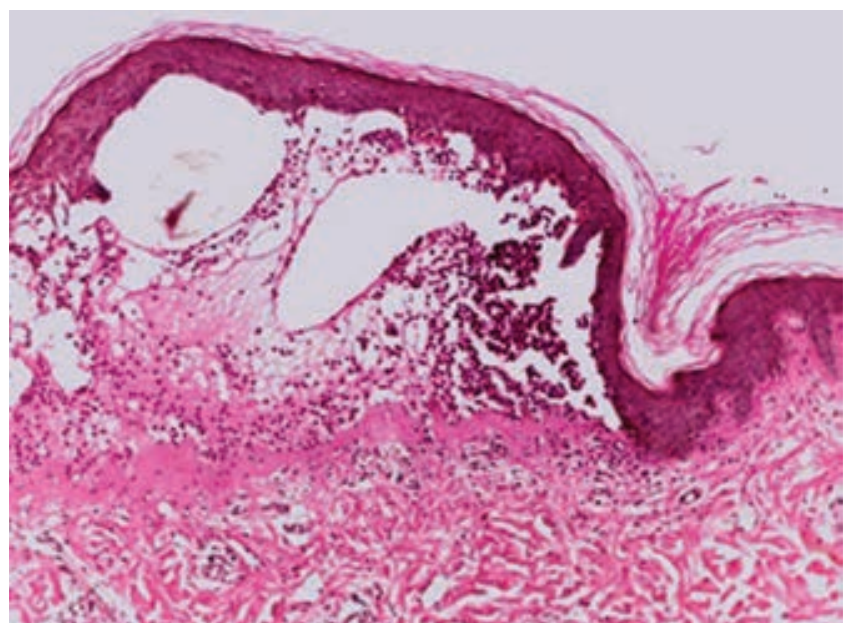

Figura 3: Ampolla subepidérmica que contiene fibrina y restos celulares (H\&E 10x).

presentando únicamente manchas residuales (Figura 6). Se le indica continuar sólo con la ingesta de dapsona $100 \mathrm{mg} /$ día, sin desarrollar efectos secundarios. Continúa en vigilancia en el Servicio de Enfermedades Ampollosas Autoinmunes del Centro Dermatológico Pascua.

\section{COMENTARIO}

Este caso en particular nos lleva a reflexionar sobre la gran variedad de manifestaciones clínicas que 
se pueden observar en las diferentes enfermedades ampollosas autoinmunes y sobre la escasa frecuencia con que se presentan algunas otras, como es el caso que nos ocupa. Por lo anterior, es de suma importancia integrar lo más pronto posible un diagnóstico correcto, teniendo como base una historia clínica lo más completa posible así como el resultado del estudio histopatológico y el de la inmunofluorescencia.

Es importante también realizar los diagnósticos diferenciales, y administrar el tratamiento adecuado lo más pronto posible por el tiempo que sea necesario.

En el caso de esta paciente, se tuvieron que prescribir dosis altas de esteroides debido a la extensión

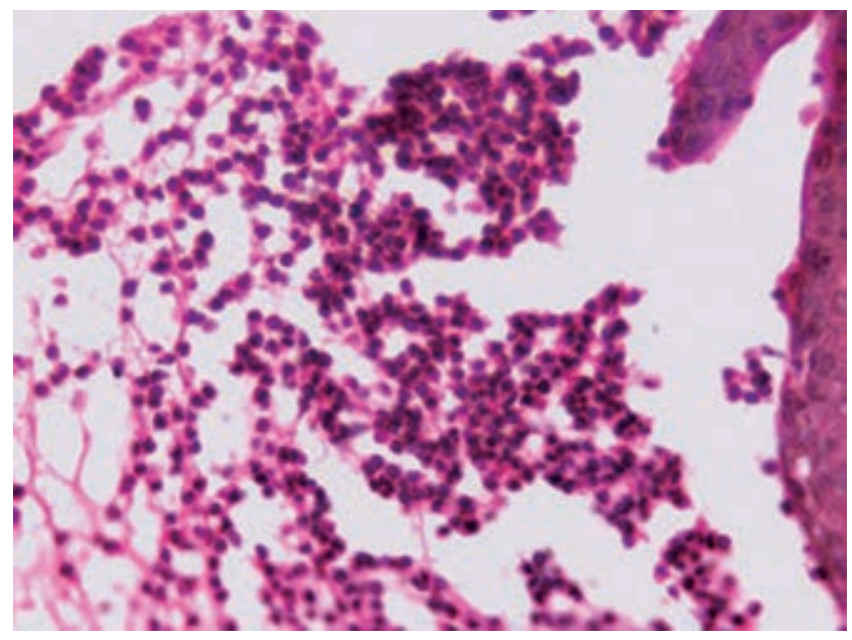

Figura 4: Contenido de la ampolla constituido principalmente por polimorfonucleares (H\&E 40x).

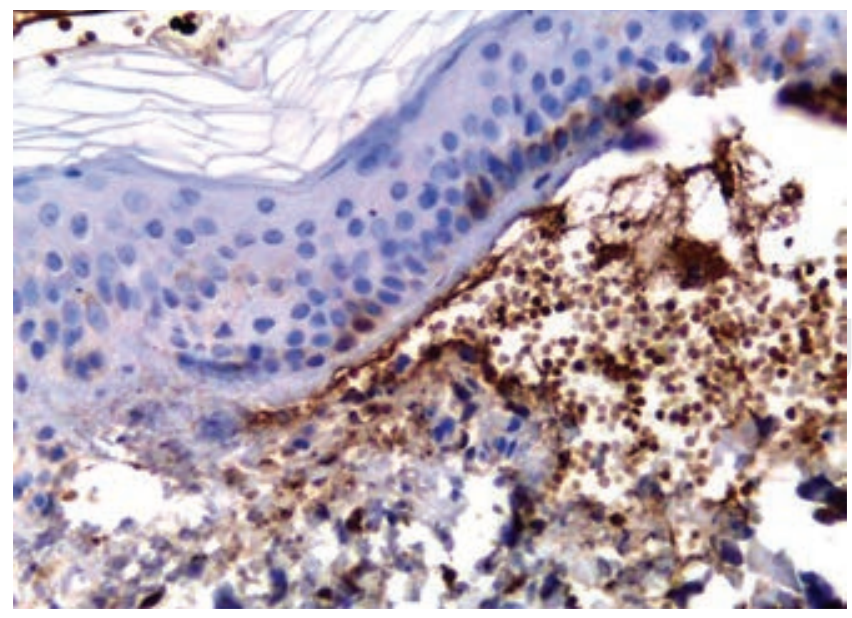

Figura 5: Inmunohistoquímica positiva para depósitos de IgA lineal.

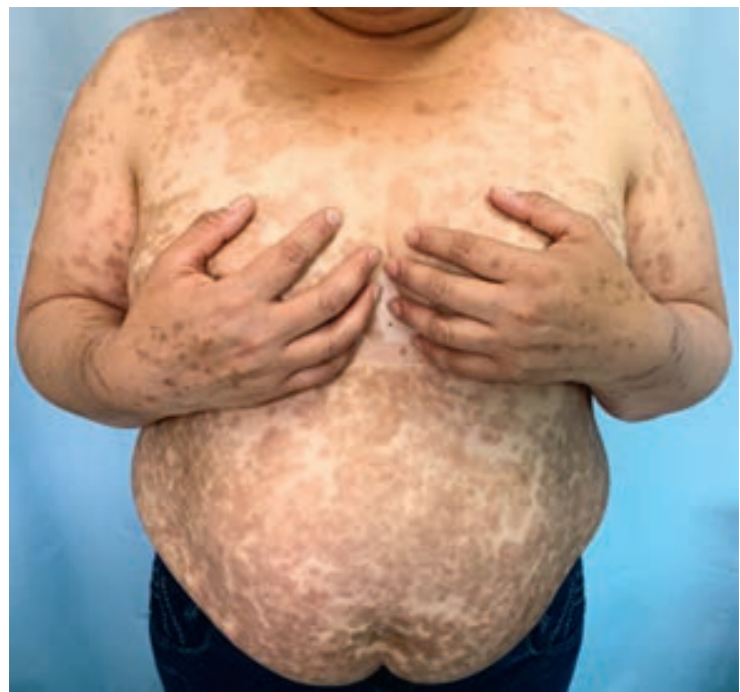

Figura 6: Evolución después de cinco meses de tratamiento. Se observan numerosas manchas residuales.

de la dermatosis, las cuales se pudieron suspender en pocos meses.

El seguimiento de estos pacientes debe ser estrecho por la posibilidad de recaídas al disminuir la dosis de los medicamentos, o por cursar con infecciones u otras comorbilidades.

\section{REFERENCIAS}

1. Chanal J, Ingen-Housz-Oro S, Ortonne N, Duong TA, Thomas M, Valeyrie-Allanore L. Linear IgA bullous dermatosis: comparison between the drug-induced and spontaneous forms. Br J Dermatol. 2013;169:1041-1048.

2. Chaudhari S, Mobini N. Linear IgA bullous dermatosis a rare clinicopathologic entity with an unusual presentation. Clin Aesthet Dermatol. 2015;8:43-46.

3. Caballero EG, Chiesura V, Hernández M, Valente E, Kurpis M, Ruiz LA. Dermatosis por depósito lineal de IgA: comunicación de tres casos y revisión de la literatura. Arch Argent Dermatol. 2014;64:7-10.

4. Bernett CN, Rosario-Collazo JA. Linear IgA dermatosis. [Updated 2019 Jun 4]. In: StatPearls [Internet]. Treasure Island (FL): StatPearls Publishing; 2019. Available in: https://europepmc.org/ books/NBK526113;jsessionid=DCA963885EF1BF994F9EFA6F5 48F1E4A.

5. Venning AV. Linear IgA disease: clinical presentation, diagnosis, and pathogenesis. Dermatol Clin. 2011;29:453-458.

6. Calonje E. McKee's pathology of the skin with clinical correlations. Vol. 1: USA: Saunders Elsevier.p. 166.

7. Leuci S, Ruoppo E, Adamo D, Calabria E, Mignogna MD. Oral autoimmune vesicobullous diseases: classification, clinical 
presentations, molecular mechanisms, diagnostic algorithms, and management. Periodontol 2000. 2019;80:77-88.

8. Fuentelsaz del Barrio V, Campos DM. Dermatosis IgA lineal de la infancia. Rev Pediatr Aten Primaria. 2013;15:141-145.

9. García-García C, Cepeda-Valdés R, Alarcón G, Gómez-Garza AA, Salas-Alanís JC. Dermatosis ampollar por depósitos de IgA lineal: reporte de un caso. Medicina Universitaria. 2013;15:135-140.
Correspondencia:

Dra. Josefina De Peña Ortiz

Dr. Vértiz Núm. 464 esq. Eje 3 Sur,

Col. Buenos Aires, 06780,

Alcaldía Cuauhtémoc, CDMX.

Tel. 5519-6351.

E-mail: jovi.60@hotmail.com 